\title{
Интерполяционная теорема для простой паранормальной логики $\operatorname{Int}_{0, \omega}{ }^{1}$
}

\author{
B. м. Попов
}

\begin{abstract}
A proof of interpolation theorem for simple paranormal logic $I n t_{0, \omega}$ is proposed.

Keywords: quasi-elementary formula, paranormal logic, interpolation theorem
\end{abstract}

Предлагается доказательство интерполяционной теоремы для простой паранормальной логики $\operatorname{Int}_{0, \omega}$.

Определяем язык $L$ как пропозициональный язык, алфавиту которого принадлежат только $\&, \vee, \supset$ (бинарные логические связки языка $L), \neg($ унарная логическая связка языка $L),),($ (технические символы языка $L$ ), $p_{1}, p_{2}, p_{3}, \ldots$ (пропозициональные переменные языка $L$ ). Принимаем сокращение «пп» для выражения «пропозициональная переменная языка $L »$. Определение $L$-формулы обычно: (1) всякая пп есть $L$-формула, $(2)$ если $A$ и $B$ есть $L$-формулы, то $(A \& B),(A \vee B),(A \supset B),(\neg A)$ есть $L$-формулы, $(3)$ ничто другое не есть $L$-формула. Условимся, что $\mathbf{N}$ есть множество всех целых положительных чисел и для всякой $L$-формулы $A W(A)$ есть множество всех пп, входящих в $A$. Квазиэлементарной $L$-формулой называем $L$-формулу, в которую не входят ни $\&$, ни $\vee$, ни $\supset$. Очевидно, что для всякой квазиэлементарной $L$-формулы $E$ число всех вхождений связки $\neg$ в $E$ есть целое неотрицательное число (называем это число длиной квазиэлементарной $L$-формулы $E$ ). Ясно, что для всякой пп $p$ и всякого $n$ из $\mathbf{N}$ существует единственная квазиэлементарная $L$-формула $A$, удовлетворяющая условию: $p$ входит в $A$ и число всех вхождений связки $\neg$ в $A$ равно $n$. Для всякой пп

\footnotetext{
${ }^{1}$ Работа выполнена при поддержке РФФИ, грант №10-06-00224а.
} 
$p$ и всякого $n$ из $\mathbf{N}$ обозначаем через $\left(\neg^{(n)} p\right)$ квазиэлементарную $L$-формулу, в которую входит $p$ и число всех вхождений связки $\neg$ в которую равно $n$. Следуя [1], определяем исчисления $H \operatorname{Int}_{0,0}$ и $\operatorname{Int}_{0, \omega}$ гильбертовского типа. Язык этих исчислений есть $L$. Множеству всех аксиом исчисления $\operatorname{Int}_{0,0}$ принадлежат все те и только те $L$-формулы, каждая из которых имеет хотя бы один из следующих видов (здесь $A, B$ и $C$ - формулы):

(I) $((A \supset B) \supset((B \supset C) \supset(A \supset C)))$,

(II) $(A \supset(A \vee B))$,

(III) $(B \supset(A \vee B))$,

(IV) $((A \supset C) \supset((B \supset C) \supset((A \vee B) \supset C)))$,

(V) $((A \& B) \supset A)$,

(VI) $((A \& B) \supset B)$,

(VII) $((C \supset A) \supset((C \supset B) \supset(C \supset(A \& B))))$,

(VIII) $((A \supset(B \supset C)) \supset((A \& B) \supset C))$,

$(\mathrm{IX})(((A \& B) \supset C) \supset(A \supset(B \supset C)))$,

$(\mathrm{X}, 0)((\neg B) \supset(B \supset A))$,

$(\mathrm{XI}, 0)((B \supset(\neg(A \supset A))) \supset(\neg B))$.

Множеству всех аксиом исчисления $\operatorname{Hnt}_{0, \omega}$ принадлежат все те и только те $L$-формулы, каждая из которых имеет вид $(\mathrm{X}, 0)$ $((\neg D) \supset(D \supset A))$ или $(\mathrm{XI}, \omega)((D \supset(\neg(A \supset A))) \supset(\neg D))$ (где $A$ есть $L$-формула, а $D$ есть $L$-формула, не являющаяся квазиэлементарной $L$-формулой), или имеет хотя бы один из видов (I)(IX). Правило modus ponens в $L$ является единственным правилом исчисления $\operatorname{HInt}_{0,0}$ и единственным правилом исчисления Int $_{0, \omega}$. Доказательства в Int $_{0,0}\left(\right.$ Int $_{0,0}$-доказательства) и доказательства в $\operatorname{HInt}_{0, \omega}\left(\right.$ Int $_{0, \omega}$-доказательства $)$ строятся обычным для исчислений гильбертовского типа образом. Стандартно определяются длина Int $_{0,0}$-доказательства, длина Int $_{0, \omega}$-доказательства, Int $_{0,0}$-доказуемая $L$-формула и $H$ Int $_{0, \omega}$-доказуемая $L$-формула. Для всякой $L$-формулы $A$ запись «HInt $t_{0,0} \vdash A »$ означает, что $A$ есть $H$ Int $_{0,0}$-доказуемая $L$-формула, а запись «HInt $t_{0, \omega} \vdash A »-$ что $A$ есть $H$ Int $_{0, \omega}$-доказуемая $L$-формула. Обозначаем множество всех $H \operatorname{Int}_{0,0}$-доказуемых $L$-формул через $I n t_{0,0}$, а множество всех $H I n t_{0, \omega}$-доказуемых $L$-формул - через $\operatorname{Int}_{0, \omega}$.

ЗАМЕЧАНИЕ. Можно доказать, что (1) Int $t_{0,0}$ и Int $t_{0, \omega}$ являются логиками в $L$ (в смысле [1]), то есть являются непустыми мно- 
жествами $L$-формул, каждое из которых замкнуто относительно modus ponens в $L$ и относительно подстановки $L$-формулы в $L$-формулу вместо пп, и (2) Int $t_{0, \omega}$ строго включается в Int $t_{0,0}$. При этом, согласно [1]: (a) Int 0,0 равно множеству всех интуиционистских тавтологий в языке $L$, то есть $I_{0,0}$ является интуиционистской логикой в языке $L$, (б) $I n t_{0, \omega}$ есть простая паранормальная логика.

Интерполяционная теорема (первоначально доказанная в [4] для классической логики предикатов) доказана в [3] для интуиционистской логики предикатов. С помощью интерполяционной теоремы для интуиционистской логики предикатов можно обосновать следующую интерполяционную теорему для интуиционистской логики в языке $L$ (то есть для Int $_{0,0}$ ).

Для всяких L-формул $A$ и В: если $(A \supset B)$ есть интуииионистская тавтология в языке $L$ u $W(A) \cap W(B) \neq \oslash$, то существует такая L-формула $C$, что $(A \supset C)$ u $(C \supset B)$ являются интуииионистскими тавтологиями в языке $L$ и $W(C) \subseteq W(A) \cap W(B)$.

Цель предлагаемой работы - доказательство интерполяционной теоремы для $\operatorname{Int}_{0, \omega}$.

TEOPЕМА (Интерполяционная теорема для Int $\left.t_{0, \omega}\right)$. Для всяких $L$-формул $A$ и В: если $(A \supset B) \in \operatorname{Int}_{0, \omega} u W(A) \cap W(B) \neq \varnothing$, то существует такая $L$-формула $C$, что $(A \supset C) \in$ Int $_{0, \omega}$, $(C \supset B) \in \operatorname{Int}_{0, \omega} u W(C) \subseteq W(A) \cap W(B)$.

Доказательство. В предлагаемом здесь доказательстве интерполяционной теоремы для $\operatorname{Int}_{0, \omega}$ использованы нижеследующие леммы (0)-(12).

Условимся, что для всякого $n$ из $\mathbf{N}$, для всяких попарно различных пп $q_{1}, \ldots, q_{n}$ и всяких $A, B_{1}, \ldots, B_{n}$, являющихся $L$-формулами, $\left[q_{1} \ldots q_{n} / B_{1} \ldots B_{n}\right](A)$ обозначает результат одновременной подстановки $L$-формул $B_{1}, \ldots, B_{n}$ в $L$-формулу $A$ вместо пп $q_{1}, \ldots, q_{n}$ соответственно.

ЛЕММА 0. Для всякого $n$ из $N$, для всяких попарно различных $n n q_{1}, \ldots, q_{n}$ и для всяких L-формул $A, B_{1}, \ldots, B_{n}$ : если Int $_{0, \omega} \vdash$ $A$, mo HInt $t_{0, \omega} \vdash\left[q_{1} \ldots q_{n} / B_{1} \ldots B_{n}\right](A)$.

Лемма 0 доказана индукцией по длине $H \operatorname{Int}_{0, \omega}$-доказательства. 
Доказана также следующая лемма 1.

ЛЕММА 1. Существует единственное отображение $\psi$ множества всех L-формул в себл, удовлетворяюоее условиям: (1) для всякой пп q верно, что $\psi(q)=q$, (2) для всякой пn $q$ верно, что $\psi((\neg q))=(\neg(q \& q))$, (3) для вслкой L-формуль $A$, не являюшейся пп, верно, что $\psi((\neg A))=(\neg \psi(A))$, (4) для всяких $L$-формул $A$ и $B$ и для всякой бинарной логической связки языка $L$ верно, что $\psi((A \bullet B))=(\psi(A) \bullet \psi(B))$.

Отображение, существование и единственность которого утверждается в лемме 1 , обозначаем через $d u b$. Опираясь на лемму 1 , можно доказать (индукцией по длине $\operatorname{HInt}_{0,0}$-доказательства) лемму 2.

ЛЕММА 2. Для всякой L-формуль A: если HInt I, $_{0} \vdash A$, mо $H \operatorname{Int}_{0, \omega} \vdash d u b(A)$.

Определение: негативно-регулярной $L$-формулой называем такую $L$-формулу $A$, в которую не входит ни одна $L$-формула вида $(\neg q)$, где $q$ есть пп.

ЛЕММА 3. Для всякой негативно-регулярной L-формуль $A$ верно, что $\operatorname{dub}(A)=A$.

Лемма 3 доказана индукцией по построению $L$-формулы.

ЛЕММА 4. Для всякой $L$-формуль $A$ верно, что $W(d u b(A))=$ $W(A)$.

Лемма 4 доказана индукцией по построению $L$-формулы.

Доказана также следующая лемма 5.

ЛЕММА 5. Пусть $F$ есть L-формула, $n \in \boldsymbol{N}, q_{1}, \ldots, q_{n}$ есть попарно различные $n n, W(F)=\left\{q_{1}, \ldots, q_{n}\right\}, k$ есть наибольшее иелое положительное число в $\{u \mid u \in N$ и существует такая $n$ n $q$, что $\left(\neg^{(u)} q\right)$ входит в $\left.F\right\}, u r_{1}^{1}, \ldots, r_{1}^{k}, \ldots, r_{n}^{1}, \ldots, r_{n}^{k}$ есть попарно различные пп, ни одна из которых не принадлежит множеству $\left\{q_{1}, \ldots, q_{n}\right\}$. Тогда существует единственное отображение $\varphi$ множества всех L-формул в себл, удовлетворяющее условиям:

(а) для всякой пп р верно, что $\varphi(p)=p$,

(b) для всякого $m$ из $\mathrm{N}$ и для всякой пn, не принадлежсащей множеству $\left\{q_{1}, \ldots, q_{n}\right\}$, верно, что $\varphi\left(\left(\neg^{(m)} p\right)\right)=\left(\neg^{(m)} p\right)$, 
(c) для всякого такого $m$ из $N$, что $m>q$, и для всякого $i$ из $\{1, \ldots, n\}$ верно, что $\varphi\left(\left(\neg^{(m)} q_{i}\right)\right)=\left(\neg \varphi\left(\left(\neg^{(m-1)} q_{i}\right)\right)\right)$,

(d) для всякого такого $m$ из $N$, что $m \leq k$, и для всякого $i$ из $\{1, \ldots, n\}$ верно что $\varphi\left(\left(\neg^{(m)} q_{i}\right)\right)=r_{i}^{m}$,

(е) для всякой $L$-формулы $A$, не являющейся квазиэлементарной $L$-формулой, верно, что $\varphi((\neg A))=(\neg \varphi(A))$,

(f) для всяких L-формул $A$ и $B$ и для всякой бинарной логической связки $\bullet$ языка $L$ верно, что $(\varphi(A \bullet B))=(\varphi(A)$ $\varphi(B))$.

ЛЕММА 6. Пусть $F$ есть $L$-формула, $n \in N, q_{1}, \ldots, q_{n}$ есть попарно различные $n n, W(F)=\left\{q_{1}, \ldots, q_{n}\right\}, k$ есть наибольшее иелое положительное число в $\{u \mid u \in N$ u существует такая $n$ n $q$, что $\left(\neg^{(u)} q\right)$ входит в $\left.F\right\}, r_{1}^{1}, \ldots, r_{1}^{k}, \ldots, r_{n}^{1}, \ldots, r_{n}^{k}$ есть попарно различные пп, ни одна из которых не принадлежит множеству $\left\{q_{1}, \ldots, q_{n}\right\}$, а $\varphi$ есть отображение множества всех $L$-формул в себл, удовлетворяющее условиям (a)-(f), сформулированным в лемме 5. Тогда для всякой L-формуль $A$ : если $\operatorname{HInt}_{0, \omega} \vdash A$, mo HInt $\operatorname{In}_{0,0} \vdash \varphi(A)$.

Лемма 6 доказана индукцией по длине $\operatorname{HInt}_{0, \omega}$-доказательства. ЛЕММА 7. В условиях леммь 6 верно, что $\varphi(A)$ есть квазиэлементарная $L$-формула для всякой квазиэлементарной $L$ формуль $A$.

Доказательство леммы 7, базирующееся на использовании определения отображения $\varphi$, тривиально.

ЛЕММА 8. В условиях леммы 6 верно, что для всякой L-формуль $A$ : если $W(A) \subseteq\left\{q_{1}, \ldots, q_{n}\right\}$ и длина всякой квазиэлементарной $L$-формуль, входящей в $A$, не больше $k$, то всякая квазиэлементарная L-формула, входлщая в $\varphi(A)$, является $n$.

Лемма 8 доказана индукцией по построению $L$-формулы.

ЛЕММА 9. В условиях леммы 6 верно, что для всякой $L$-формулы $A$ : если $W(A) \subseteq\left\{q_{1}, \ldots, q_{n}\right\}$ и длина всякой квазиэлементарной $L$-формуль, входящей в $A$, не больше $k$, то $\left[r_{1}^{1} \ldots r_{1}^{k} \ldots\right.$ $\left.\left.r_{n}^{1} \ldots r_{n}^{k} /\left(\neg^{(1}\right) q_{1}\right) \ldots\left(\neg^{(k)} q_{1}\right) \ldots\left(\neg^{(1)} q_{n}\right) \ldots\left(\neg^{(k)} q_{n}\right)\right](\varphi(A))=A$.

Лемма 9 доказана индукцией по построению $L$-формулы. 
ЛЕММА 10. В условиях леммы 6 верно, что для всякой $L$ формуль $A$ : если $W(A) \subseteq\left\{q_{1}, \ldots, q_{n}\right\}$, длина всякой квазиэлементарной $L$-формуль, входящей в $A$, не больше $k$ u $\operatorname{HInt}_{0,0} \vdash$ $\varphi(A)$, mo HInt $\operatorname{In}_{0, \omega} \vdash A$.

Лемма 10 доказана с использованием лемм $0,2,3,8$ и 9.

ЛЕММА 11. Для всяких L-формул $A$ и В: если $(A \supset B)$ есть интуичионистская тавтология в языке $L$ u $W(A) \cap W(B) \neq \varnothing$, то существует такая $L$-формула $C$, что выполняются следующие условия: (а) $(A \supset C)$ и $(C \supset B)$ являются интуииионистскими тавтологиями в языке $L$, (б) $W(C) \subseteq W(A) \cap$ $W(B)$, (в) C есть негативно-регулярная L-формула.

Доказательство леммы 11 проведено с использованием (1) интерполяционной теоремы для интуиционистской логики в языке $L$ (формулировка этой теоремы приведена выше), (2) утверждения о том, что для всякой пп $q L$-формула $((q \supset(q \& q)) \&((q \& q) \supset$ $q))$ является интуиционистской тавтологией в языке $L$, и (3) следующей теоремы об эквивалентной замене: если $A$ и $B$ есть такие $L$-формулы, что $((A \supset B) \&(B \supset A))$ есть интуиционистская тавтология в $L$, то для всякой $L$-формулы $M$, в которую входит $A$, интуиционистской тавтологией в языке $L$ является $\left(\left(M \supset M^{\prime}\right) \&\left(M^{\prime} \supset M\right)\right)$, где $M^{\prime}$ есть результат замены в $M$ некоторых вхождений $L$-формулы $A$ вхождениями $L$-формулы $B$.

ЛЕММА 12. В условиях леммы 6 верно, что для всякой $L$ формуль $A$, входящей в $F, \varphi(A)$ есть негативно-регулярная L-формула.

\section{Доказательство.}

Лемма 12 вытекает из нижеследующих подлеммы 1 и подлеммы 2.

ПОДЛЕММА 1. В условиях леммы 6 верно, что: (1) всякая пп $p$, входящая в $F$, такова, что $\varphi(p)$ есть негативно-регулярная $L$ формула, (2) всякие $L$-формулы $A$ и $B$, входящие в $F$, таковы, что если $\varphi(A)$ и $\varphi(B)$ есть негативно-регулярные $L$-формулы и $(A \& B)$ входит в $F$, то $\varphi((A \& B))$ есть негативно-регулярная $L$ формула, (3) всякие $L$-формулы $A$ и $B$, входящие в $F$, таковы, что если $\varphi(A)$ и $\varphi(B)$ есть негативно-регулярные $L$-формулы и 
$(A \vee B)$ входит в $F$, то $\varphi((A \vee B))$ есть негативно-регулярная $L$ формула, (4) всякие $L$-формулы $A$ и $B$, входящие в $F$, таковы, что если $\varphi(A)$ и $\varphi(B)$ есть негативно-регулярные $L$-формулы и $(A \supset B)$ входит в $F$, то $\varphi((A \supset B))$ есть негативно-регулярная $L$-формула, (5) всякая $L$-формула $A$, входящая в $F$, такова, что если $\varphi(A)$ есть негативно-регулярная $L$-формула и $(\neg A)$ входит в $F$, то $\varphi((\neg A))$ есть негативно-регулярная $L$-формула.

ПОДЛЕММА 2. Для всякой $L$-формулы $F$ и для всякого множества $P L$-формул: если (1) всякая пп, входящая в $F$, принадлежит $P,(2)$ всякие $L$-формулы $A$ и $B$, входящие в $F$, таковы, что если они принадлежат $P$ и $(A \& B)$ входит в $F$, то $(A \& B) \in P$, (3) всякие $L$-формулы $A$ и $B$, входящие в $F$, таковы, что если они принадлежат $P$ и $(A \vee B)$ входит в $F$, то $(A \vee B) \in P$, (4) всякие $L$-формулы $A$ и $B$, входящие в $F$, таковы, что если они принадлежат $P$ и $(A \supset B)$ входит в $F$, то $(A \supset B) \in P$, и (5) всякая $L$-формула $A$, входящая в $F$, такова, что если $A \in P$ и $(\neg A)$ входит в $F$, то $(\neg A) \in P$, то всякая $L$-формула, входящая в $F$, принадлежит $P$.

Q.E.D.

Доказательство интерполяционной теоремы для $\operatorname{Int}_{0, \omega}$.

(1) $A_{0}$ и $B_{0}$ есть $L$-формулы (допущение).

(2) $\left(A_{0} \supset B_{0}\right) \in \operatorname{Int}_{0, \omega}$ и $W\left(A_{0}\right) \cap W\left(B_{0}\right) \neq \varnothing$ (допущение). Индукцией по построению $L$-формулы можно доказать, что

(3) для всякой $L$-формулы $G$ верно, что $W(G)$ есть непустое конечное множество пп.

(4) $\left(A_{0} \supset B_{0}\right)$ есть $L$-формула (из $(1)$, по определению $L$ формулы).

(5) $W\left(\left(A_{0} \supset B_{0}\right)\right)$ есть непустое конечное множество пп (из (3) и (4)). Пусть

(6) $n \in \mathbf{N}, q_{1}, \ldots, q_{n}$ есть попарно различные пп, $W((A \supset$ $B))=\left\{q_{1}, \ldots, q_{n}\right\}$. Допустим, что

(7) не существует такое $u$ из $\mathbf{N}$, что для некоторой пп $p\left(\neg^{(u)} p\right)$ входит в $\left(A_{0} \supset B_{0}\right)$.

(8) $\left(A_{0} \supset B_{0}\right) \in \operatorname{Int}_{0,0}$ (из того, что $\left(A_{0} \supset B_{0}\right) \in \operatorname{Int}_{0, \omega}$ (см. $(2))$, и того, что $I_{0, \omega} \subseteq \operatorname{Int}_{0,0}$ (см. замечание)).

(9) Существует такая $L$-формула $C$, что $\left(\left(A_{0} \supset C\right) \in\right.$ Int $_{0,0}$, $\left(C \supset B_{0}\right) \in$ Int $_{0,0}$ и $W(C) \subseteq W\left(A_{0}\right) \cap W\left(B_{0}\right)$ (из (1) и того, что 
$W\left(A_{0}\right) \cap W\left(B_{0}\right) \neq \varnothing$ (см. (2)), по интерполяционной теореме для Int $\left._{0,0}\right)$. Пусть

(10) $C_{0}$ есть $L$-формула, $\left(A_{0} \supset C_{0}\right) \in \operatorname{Int}_{0,0},\left(C_{0} \supset B_{0}\right) \in \operatorname{Int}_{0,0}$ и $W\left(C_{0}\right) \subseteq W\left(A_{0}\right) \cap W\left(B_{0}\right)$. Опираясь на (10) и определение множества Int $t_{0,0}$, получаем, что

(11) $\operatorname{HInt}_{0,0} \vdash\left(A_{0} \supset C_{0}\right)$ и $\operatorname{HInt}_{0,0} \vdash\left(C_{0} \supset B_{0}\right)$.

(12) $\operatorname{HInt}_{0, \omega} \vdash d u b\left(\left(A_{0} \supset C_{0}\right)\right)$ и $\operatorname{HInt}_{0, \omega} \vdash d u b\left(\left(C_{0} \supset B_{0}\right)\right)$ (из (11) и того, что $\left(A_{0} \supset C_{0}\right)$ и $\left(C_{0} \supset B_{0}\right)$ есть $L$-формулы, по лемме 2).

(13) $\operatorname{HInt}_{0, \omega} \vdash\left(d u b\left(A_{0}\right) \supset d u b\left(C_{0}\right)\right)$ и $\operatorname{HInt}_{0, \omega} \vdash\left(d u b\left(C_{0}\right) \supset\right.$ $\left.d u b\left(B_{0}\right)\right)$ (из (12), по определению $d u b$ и по лемме 1$)$. В свете (7) ясно, что

(14) не существует такое $u$ из $\mathbf{N}$, что для некоторой пп $p$ $\left(\neg^{(u)} p\right)$ входит в $A_{0}$, и не существует такое $u$ из $\mathbf{N}$, что для некоторой пп $p\left(\neg^{(u)} p\right)$ входит в $B_{0}$.

$(15) d u b\left(A_{0}\right)=A_{0}$ и $d u b\left(B_{0}\right)=B_{0}$ (из (1) и (14), по лемме 3).

(16) $\operatorname{HInt}_{0, \omega} \vdash\left(A_{0} \supset d u b\left(C_{0}\right)\right)$ и $\operatorname{HInt}_{0, \omega} \vdash\left(d u b\left(C_{0}\right) \supset B_{0}\right)$ (из (13) и (15)).

(17) $W\left(\operatorname{dub}\left(C_{0}\right)\right)=W\left(C_{0}\right)$ (из того, что $\mathrm{C}_{0}$ есть $L$-формула (см. (10)), по лемме 4).

(18) $W\left(d u b\left(C_{0}\right)\right) \subseteq W\left(A_{0}\right) \cap W\left(B_{0}\right)$ (из того, что $W\left(C_{0}\right) \subseteq$ $W\left(A_{0}\right) \cap W\left(B_{0}\right)$ (см. (10)), и (17)).

(19) Существует такая $L$-формула $C$, что $H \operatorname{Int}_{0, \omega} \vdash\left(A_{0} \supset C\right)$, $H_{\text {Int }_{0, \omega}} \vdash\left(C \supset B_{0}\right)$ и $W(C) \subseteq W\left(A_{0}\right) \cap W\left(B_{0}\right)$ ( из (16), (18) и того, что $d u b\left(C_{0}\right)$ есть $L$-формула). Снимая допущение $(7)$, получаем, что

(20) если не существует такое $u$ из $\mathbf{N}$, что для некоторого $i$ из $\{1, \ldots, n\}\left(\neg^{(u)} q_{i}\right)$ входит в $\left(A_{0} \supset B_{0}\right)$, то существует такая $L$-формула $C$, что Int $_{0, \omega} \vdash\left(A_{0} \supset C\right)$, HInt $t_{0, \omega} \vdash\left(C \supset B_{0}\right)$ и $W(C) \subseteq W\left(A_{0}\right) \cap W\left(B_{0}\right)$. Допустим, что

(21) существует такое $u$ из $\mathbf{N}$, что для некоторой пп $p\left(\neg^{(u)} p\right)$ входит в $\left(A_{0} \supset B_{0}\right)$.

(22) $\left\{u \mid u \in \mathbf{N}\right.$ и существует такая пп $p$, что $\left(\neg^{(u)} p\right)$ входит в $\left.\left(A_{0} \supset B_{0}\right)\right\} \neq \varnothing$ (из (21)). Ясно, что

(23) $\left\{u \mid u \in \mathbf{N}\right.$ и существует такая пп $p$, что $\left(\neg^{(u)} p\right)$ входит в $\left.\left(A_{0} \supset B_{0}\right)\right\}$ есть конечное множество. Опираясь на $(22)$ и $(23)$, получаем, что

(24) в $\left\{u \mid u \in \mathbf{N}\right.$ и существует такая пп $p$, что $\left(\neg^{(u)} p\right)$ входит в 
$\left.\left(A_{0} \supset B_{0}\right)\right\}$ существует наибольшее целое положительное число. Пусть

(25) $k$ есть наибольшее целое положительное число в $\{u \mid u \in$ $\mathbf{N}$ и существует такая пп $p$, что $\left(\neg^{(u)} p\right)$ входит в $\left.\left(A_{0} \supset B_{0}\right)\right\}$. Учитывая, что множество $\left\{q_{1}, \ldots, q_{n}\right\}$ конечно, а множество всех пп бесконечно, положим, что

(26) $r_{1}^{1}, \ldots, r_{1}^{k}, \ldots, r_{n}^{1}, \ldots, r_{n}^{k}$ есть попарно различные пп, ни одна из которых не принадлежит множеству $\left\{q_{1}, \ldots, q_{n}\right\}$.

(27) Существует единственное отображение $\varphi$ множества всех $L$-формул в себя, удовлетворяющее условиям (a), (b), (c), (d), (е) и (f), сформулированным в лемме 5 (из (4), (6), (25) и (26), по лемме 5). Пусть

(28) $\varphi_{0}$ есть отображение множества всех $L$-формул в себя, удовлетворяющее условиям: $\left(\mathrm{a}^{\prime}\right)$ для всякой пп $p$ верно, что $\varphi_{0}(p)=p,\left(\mathrm{~b}^{\prime}\right)$ для всякого $m$ из $\mathbf{N}$ и для всякой пп, не принадлежащей множеству $\left\{q_{1}, \ldots, q_{n}\right\}$, верно, что $\varphi_{0}\left(\left(\neg^{(m)} p\right)\right)=$ $\left(\neg^{(m)} p\right),\left(\mathrm{c}^{\prime}\right)$ для всякого такого $m$ из $\mathbf{N}$, что $m>k$, и для всякого $i$ из $\{1, \ldots, n\}$ верно, что $\varphi_{0}\left(\left(\neg^{(m)} q_{i}\right)\right)=\left(\neg \varphi_{0}\left(\left(\neg^{(m)} q_{i}\right)\right)\right)$, $\left(\mathrm{d}^{\prime}\right)$ для всякого такого $m$ из $\mathbf{N}$, что $m \leq k$, и для всякого $i$ из $\{1, \ldots, n\}$ верно, что $\varphi_{0}\left(\left(\neg^{(m)} q_{i}\right)\right)=r_{i}^{m},\left(\mathrm{e}^{\prime}\right)$ для всякой $L$ формулы $A$, не являющейся квазиэлементарной $L$-формулой, верно, что $\varphi_{0}((\neg A))=\left(\neg \varphi_{0}(A)\right),\left(\mathrm{f}^{\prime}\right)$ для всяких $L$-формул $A$ и $B$ и для всякой бинарной логической связки • языка $L$ верно, что $\varphi_{0}((A \bullet B))=\left(\varphi_{0}(A) \bullet \varphi_{0}(B)\right)$.

(29) $\operatorname{H~Int~}_{0,0} \vdash \varphi_{0}\left(\left(A_{0} \supset B_{0}\right)\right)$ (из (4), (6), (25), (26) и (28), по лемме 6$)$.

(30) $\varphi_{0}\left(\left(A_{0} \supset B_{0}\right)\right)=\left(\varphi_{0}\left(A_{0}\right) \supset \varphi_{0}\left(B_{0}\right)\right)$ (из (1) и пункта $\left(\mathrm{f}^{\prime}\right)$ утверждения (28)).

(31) H Int $_{0,0} \vdash\left(\varphi_{0}\left(A_{0}\right) \supset \varphi_{0}\left(B_{0}\right)\right)$ (из $(29)$ и $\left.(30)\right)$.

(32) Если $W\left(\varphi_{0}\left(A_{0}\right)\right) \cap W\left(\varphi_{0}\left(B_{0}\right)\right)=\varnothing$, то существует такая $L$-формула $C$, что Int $_{0, \omega} \vdash\left(A_{0} \supset C\right)$, HInt I,$\omega \vdash\left(C \supset B_{0}\right)$ и $W(C) \subseteq W\left(A_{0}\right) \cap W\left(B_{0}\right)$.

Докажем утверждение (32).

(32.1) $W\left(\varphi_{0}\left(A_{0}\right)\right) \cap W\left(\varphi_{0}\left(B_{0}\right)\right)=\varnothing$ (допущение). Известно, что верно следующее утверждение (32.2).

(32.2) Для всяких $L$-формул $A$ и $B$ : если $(A \supset B)$ есть интуиционистская тавтология в $L$ и $W(A) \cap W(B)=\varnothing$, то $(\neg A)$ есть интуиционистская тавтология в $L$ или $B$ есть интуиционистская 
тавтология в $L$. Опираясь на утверждения (31), (32.1) и (32.2), получаем, что

$(32.3)\left(\neg \varphi_{0}\left(A_{0}\right)\right)$ есть интуиционистская тавтология в $L$ или $\left(\varphi_{0}\left(B_{0}\right)\right)$ есть интуиционистская тавтология в $L$.

(32.4) $\left(\neg \varphi_{0}\left(A_{0}\right)\right)$ есть интуиционистская тавтология в $L$ (допущение). Отсюда и из того, что ни одна интуиционистская тавтология в $L$ не является квазиэлементарной $L$-формулой, получаем, что

$(32.5)\left(\neg \varphi_{0}\left(A_{0}\right)\right)$ не есть квазиэлементарная $L$-формула.

(32.6) $\varphi_{0}\left(A_{0}\right)$ не есть квазиэлементарная $L$-формула (из (32.5), по определению квазиэлементарной $L$-формулы).

(32.7) $A_{0}$ не есть квазиэлементарная $L$-формула (из (1), (4), $(6),(25),(26),(28)$ и $(32.6)$, по лемме 7).

$(32.8) \varphi_{0}\left(\left(\neg A_{0}\right)\right)=\left(\neg \varphi_{0}\left(A_{0}\right)\right)$ (из $(1),(32.7)$ и пункта $\left(\mathrm{c}^{\prime}\right)$ утверждения (28)).

$(32.9) \varphi_{0}\left(\left(\neg A_{0}\right)\right)$ есть интуиционистская тавтология в $L$ (из (32.4) и (32.8)). В свете утверждения (6) ясно, что

(32.10) $W\left(\left(\neg A_{0}\right)\right) \subseteq\left\{q_{1}, \ldots, q_{n}\right\}$. Опираясь на $(25)$ и (32.7), получаем, что

(32.11) длина всякой квазиэлементарной $L$-формулы, входящей в $\left(\neg A_{0}\right)$, не больше $k$.

(32.12) Int $_{0, \omega} \vdash\left(\neg A_{0}\right)$ (из того, что $\left(\neg A_{0}\right)$ есть $L$-формула, (4), (6), (25), (26), (28), (32.9), (32.10) и (32.11), по лемме 10). Вспомнив, что $W\left(A_{0}\right) \cap W\left(B_{0}\right)=\varnothing$, положим, что

(32.13) $x \in W\left(A_{0}\right) \cap W\left(B_{0}\right)$. Понятно, что тогда

(32.14) $x$ есть пропозициональная переменная языка $L$ и

$(32.15)(\neg(x \supset x))$ есть $L$-формула. Используя утверждение (1), (32.7), (32.15), определение $L$-формулы и определение аксиомы исчисления $\operatorname{HInt}_{0, \omega}$, получаем, что

(32.16) $\left(\left(\neg A_{0}\right) \supset\left(A_{0} \supset(\neg(x \supset x))\right)\right)$ есть аксиома исчисления $H \operatorname{Int}_{0, \omega}$. Но тогда ясно, что

(32.17) H Int $_{0, \omega} \vdash\left(\left(\neg A_{0}\right) \supset\left(A_{0} \supset(\neg(x \supset x))\right)\right)$. Опираясь на утверждения (32.12) и (32.17), получаем, что

(32.18) H Int $_{0, \omega} \vdash\left(A_{0} \supset(\neg(x \supset x))\right)$. Простое доказательство нижеследующего утверждения (32.19) не приводим.

(32.19) $\operatorname{HInt}_{0, \omega} \vdash\left((\neg(x \supset x)) \supset B_{0}\right)$.

$(32.20) W((\neg(x \supset x)))=\{x\}$ (из (32.14) и (32.15), по определению $W)$. 
(32.21) Существует такая $L$-формула $C$, что: $H \operatorname{Int} t_{0, \omega} \vdash\left(A_{0} \supset\right.$ $C)$, Int $_{0, \omega} \vdash\left(C \supset B_{0}\right)$ и $W(C) \subseteq W\left(A_{0}\right) \cap W\left(B_{0}\right)$ (из (32.13), (32.15), (32.18), (32.19) и (32.20)). Снимая допущение (32.4), получаем, что

(32.22) если $\left(\neg \varphi_{0}\left(A_{0}\right)\right)$ есть интуиционистская тавтология в $L$, то существует такая $L$-формула $C$, что: $\operatorname{Int}_{0, \omega} \vdash\left(A_{0} \supset C\right)$, $H$ Int $_{0, \omega} \vdash\left(C \supset B_{0}\right)$ и $W(C) \subseteq W\left(A_{0}\right) \cap W\left(B_{0}\right)$.

$(32.23) \varphi_{0}\left(B_{0}\right)$ есть интуиционистская тавтология в $L$ (допущение). В свете утверждения (6) очевидно, что

$(32.24) W\left(\varphi_{0}\left(B_{0}\right)\right) \subseteq\left\{q_{1}, \ldots, q_{n}\right\}$. Опираясь на утверждение (25), получаем, что

(32.25) длина всякой квазиэлементарной $L$-формулы, входящей в $B_{0}$, не больше $k$.

(32.26) $\operatorname{HInt}_{0, \omega} \vdash B_{0}$ (из (1), (4), (16), (25), (26), (28), (32.23), $(32.24)$ и (32. 25) по лемме 10). Учитывая, что $W\left(A_{0}\right) \cap W\left(B_{0}\right) \neq$ $\varnothing$, положим, что

(32.27) y $\in W\left(A_{0}\right) \cap W\left(B_{0}\right)$. Разумеется, тогда

(32.28) y есть пп и

(32.29) ( $(y \supset y)$ есть $L$-формула. Опираясь на утверждения (32.26) и (32. 29), нетрудно доказать, что

(32.30) Int $_{0, \omega} \vdash\left((y \supset y) \supset B_{0}\right)$. Можно доказать также, что

(32.31) HInt $_{0, \omega} \vdash\left(A_{0} \supset(y \supset y)\right)$.

(32.32) $W((y \supset y))=\{y\}$ (из (32.28) и (32.29), по определению $W)$.

(32.33) Существует такая $L$-формула $C$, что: $H \operatorname{Int} t_{0, \omega} \vdash\left(A_{0} \supset\right.$ $C)$, Int $_{0, \omega} \vdash\left(C \supset B_{0}\right)$ и $W(C) \subseteq W\left(A_{0}\right) \cap W\left(B_{0}\right)$ (из (32.27), $(32.29),(32.30),(32.31)$ и (32.32)). Снимая допущение (32.23), получаем, что

(32.34) если $\varphi_{0}\left(B_{0}\right)$ есть интуиционистская тавтология в $L$, то существует такая $L$-формула $C$, что: $H \operatorname{Int}_{0, \omega} \vdash\left(A_{0} \supset C\right)$, HInt $_{0, \omega} \vdash\left(C \supset B_{0}\right)$ и $W(C) \subseteq W\left(A_{0}\right) \cap W\left(B_{0}\right)$.

(32.35) Существует такая $L$-формула $C$, что: $H \operatorname{Int}_{0, \omega} \vdash\left(A_{0} \supset\right.$ $C)$, Int $_{0, \omega} \vdash\left(C \supset B_{0}\right)$ и $W(C) \subseteq W\left(A_{0}\right) \cap W\left(B_{0}\right)$ (из (32.3), $(32.23)$ и (32.34)). Снимая допущение (32.1), получаем, что если $W\left(\varphi_{0}\left(A_{0}\right)\right) \cap W\left(\varphi_{0}\left(B_{0}\right)\right)=\varnothing$, то существует такая $L$-формула $C$, что: $\operatorname{HInt}_{0, \omega} \vdash\left(A_{0} \supset C\right), \operatorname{HInt}_{0, \omega} \vdash\left(C \supset B_{0}\right)$ и $W(C) \subseteq$ $W\left(A_{0}\right) \cap W\left(B_{0}\right)$.

Утверждение (32) доказано. 
(33) Если $W\left(\varphi_{0}\left(A_{0}\right)\right) \cap W\left(\varphi_{0}\left(B_{0}\right)\right) \neq \varnothing$, то существует такая $L$-формула $C$, что: Int $_{0, \omega} \vdash\left(A_{0} \supset C\right)$, HInt $\operatorname{In}_{0, \omega} \vdash\left(C \supset B_{0}\right)$ и $W(C) \subseteq W\left(A_{0}\right) \cap W\left(B_{0}\right)$.

Докажем утверждение (33).

(33.1) $W\left(\varphi_{0}\left(A_{0}\right)\right) \cap W\left(\varphi_{0}\left(B_{0}\right)\right) \neq \varnothing$ (допущение). Ясно, что

$(33.2) \varphi_{0}\left(A_{0}\right)$ и $\varphi_{0}\left(B_{0}\right)$ являются $L$-формулами.

(33.3) $\left(\varphi_{0}\left(A_{0}\right) \supset \varphi_{0}\left(B_{0}\right)\right) \in \operatorname{Int}_{0,0}$ (из 31), по определению множества Int $\left.t_{0,0}\right)$.

(33.4) $\left(\varphi_{0}\left(A_{0}\right) \supset \varphi_{0}\left(B_{0}\right)\right)$ есть интуиционистская тавтология в языке $L$ (из (33.3) и того, что $I_{n, 0}$ есть множество всех интуиционистских тавтологий в языке $L)$.

(33.5) Существует такая $L$-формула $C$, что: $\left(\varphi_{0}\left(A_{0}\right) \supset C\right)$ и $\left(C \supset \varphi_{0}\left(B_{0}\right)\right)$ есть интуиционистские тавтологии в $L, W(C) \subseteq$ $W\left(\varphi_{0}\left(A_{0}\right)\right) \cap W\left(\varphi_{0}\left(B_{0}\right)\right)$ и $C$ есть негативно-регулярная $L$-формула (из (33.1), (33.2) и (33.4), по лемме 11). Пусть

(33.6) $C_{0}$ есть $L$-формула, $\left(\varphi_{0}\left(A_{0}\right) \supset C_{0}\right)$ и $\left(C_{0} \supset \varphi_{0}\left(B_{0}\right)\right)$ есть интуиционистские тавтологии в $L, W\left(C_{0}\right) \subseteq W\left(\varphi_{0}\left(A_{0}\right)\right) \cap$ $W\left(\varphi_{0}\left(B_{0}\right)\right)$ и $C$ есть негативно-регулярная $L$-формула.

$(33.7)\left(\varphi_{0}\left(A_{0}\right) \supset C_{0}\right) \in \operatorname{Int}_{0,0}$ и $\left(C_{0} \supset \varphi_{0}\left(B_{0}\right)\right) \in \operatorname{Int}_{0,0}$ (из (33.6) и того, что Int $t_{0,0}$ есть множество всех интуиционистских тавтологий в языке $L)$.

(33.8) $\left(\varphi_{0}\left(A_{0}\right) \supset C_{0}\right)$ и $\left(C_{0} \supset \varphi_{0}\left(B_{0}\right)\right)$ являются $H$ Int $_{0,0}$-доказуемыми $L$-формулами (из (33.7), по соглашению об обозначении).

(33.9) $\operatorname{HInt}_{0,0} \vdash\left(\varphi_{0}\left(A_{0}\right) \supset C_{0}\right)$ и $\operatorname{HInt}_{0,0} \vdash\left(C_{0} \supset \varphi_{0}\left(B_{0}\right)\right)$ (из (33.8), по соглашению об использовании «H Int $\left.t_{0,0} \vdash \gg\right)$.

(33.10) $\operatorname{HInt}_{0, \omega} \vdash d u b\left(\left(\varphi_{0}\left(A_{0}\right) \supset C_{0}\right)\right)$ и $\operatorname{HInt}_{0, \omega} \vdash d u b\left(\left(C_{0} \supset\right.\right.$ $\left.\left.\varphi_{0}\left(B_{0}\right)\right)\right)$ (из (33.9), по лемме 2).

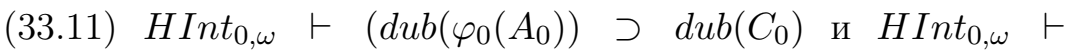
$\left(d u b\left(C_{0}\right) \supset d u b\left(\varphi_{0}\left(B_{0}\right)\right)\right)$ (из (33.10), по лемме 1$)$.

(33.12) $d u b\left(C_{0}\right)=C_{0}$ (из(33.6), по лемме 3). Очевидно, что

(33.13) $L$-формулы $A_{0}$ и $B_{0}$ входят в $L$-формулу $\left(A_{0} \supset B_{0}\right)$.

$(33.14) \varphi_{0}\left(A_{0}\right)$ и $\varphi_{0}\left(B_{0}\right)$ есть негативно-регулярные $L$-формулы (из $(1),(4),(6),(25),(26),(28)$ и (33.13), по лемме 12$)$.

$(33.15) d u b\left(\varphi_{0}\left(A_{0}\right)\right)=\varphi_{0}\left(A_{0}\right)$ и $d u b\left(\varphi_{0}\left(B_{0}\right)\right)=\varphi_{0}\left(B_{0}\right)($ из $(33.14)$, по лемме 3$)$.

(33.16) $\operatorname{HInt}_{0, \omega} \vdash\left(\varphi_{0}\left(A_{0}\right) \supset C_{0}\right)$ и $\operatorname{HInt}_{0, \omega} \vdash\left(C_{0} \supset \varphi_{0}\left(B_{0}\right)\right)$ (из $(33.11),(33.12)$ и $(33.15))$. Понятно, что 
(33.17) произведение $k$ на $n$ есть целое положительное число, $r_{1}^{1}, \ldots, r_{1}^{k}, \ldots, r_{n}^{1}, \ldots, r_{n}^{k}$ есть попарно различные пп, а $\left(\neg^{(1)} q_{1}\right), \ldots,\left(\neg^{(k)} q_{1}\right), \ldots,\left(\neg^{(1)} q_{n}\right), \ldots,\left(\neg^{(k)} q_{n}\right)$ есть $L$-формулы. Условившись вместо « $\left[r_{1}^{1} \ldots r_{1}^{k} \ldots r_{n}^{1} \ldots r_{n}^{k} /\left(\neg^{(1)} q_{1}\right) \ldots\left(\neg^{(k)} q_{1}\right) \ldots\right.$ $\left.\left(\neg^{(1)} q_{n}\right) \ldots\left(\neg^{(k)} q_{n}\right)\right] »$ использовать «S» и опираясь на $(33.16)$ и (33.17), получаем по лемме 0, что

(33.18) HInt $_{0, \omega} \vdash S\left(\left(\varphi_{0}\left(A_{0}\right) \supset C_{0}\right)\right)$ и $\operatorname{HInt}_{0, \omega} \vdash S\left(\left(C_{0} \supset\right.\right.$ $\left.\varphi_{0}\left(B_{0}\right)\right)$ ). Используя дистрибутивность $S$ относительно импликации $\supset$, получаем, что

(33.19) $L$-формула $S\left(\left(\varphi_{0}\left(A_{0}\right)\right) \quad \supset C_{0}\right)$ есть $L$-формула $\left(S\left(\varphi_{0}\left(A_{0}\right)\right) \supset S\left(C_{0}\right)\right)$, а $L$-формула $S\left(\left(C_{0} \supset \varphi_{0}\left(B_{0}\right)\right)\right)$ есть $L$ формула $\left(S\left(C_{0}\right) \supset S\left(\varphi_{0}\left(B_{0}\right)\right)\right)$.

$(33.20) \operatorname{HInt}_{0, \omega} \vdash\left(S\left(\varphi_{0}\left(A_{0}\right)\right) \supset S\left(C_{0}\right)\right)$ и $\operatorname{HInt}_{0, \omega} \vdash\left(S\left(C_{0}\right) \supset\right.$ $\left.S\left(\varphi_{0}\left(B_{0}\right)\right)\right)$ (из $(33.18)$ и (33.19), по соглашению об использовании $\ll S \gg)$. В свете утверждений (6) и $(25)$ ясно, что

(33.21) $W\left(A_{0}\right) \subseteq\left\{q_{1}, \ldots, q_{n}\right\}, W\left(B_{0}\right) \subseteq\left\{q_{1}, \ldots, q_{n}\right\}$, длина всякой квазиэлементарной $L$-формулы, входящей в $A_{0}$, не больше $k$, длина всякой квазиэлементарной $L$-формулы, входящей в $B_{0}$, не больше $k$.

(33.22) $S\left(\varphi_{0}\left(A_{0}\right)\right)=A_{0}$ и $S\left(\varphi_{0}\left(B_{0}\right)\right)=B_{0}$ (из (4), (6), (25), $(26),(28)$ и $(33.21)$, по лемме 9).

$(33.23) \operatorname{HInt}_{0, \omega} \vdash\left(A_{0} \supset S\left(C_{0}\right)\right)$ и $H \operatorname{Int}_{0, \omega} \vdash\left(S\left(C_{0}\right) \supset B_{0}\right)$ (из (33.20) и (33.22)).

(33.24) $W\left(C_{0}\right) \subseteq W\left(\varphi_{0}\left(A_{0}\right)\right) \cap W\left(\varphi_{0}\left(B_{0}\right)\right)($ из $(33.6))$.

$(33.25) W\left(C_{0}\right) \subseteq W\left(\varphi_{0}\left(A_{0}\right)\right)$ и $W\left(C_{0}\right) \subseteq W\left(\varphi_{0}\left(B_{0}\right)\right)$ (из (33.24)). Очевидно следующее утверждение

(33.26), имеющее семиотический характер. (33.26) Для всякого $m$ из $\mathbf{N}$, для всяких $L$-формул $A, B, C_{1}, \ldots, C_{m}$ и для всяких попарно различных пп $s_{1}, \ldots, s_{m}$ : если $W(A) \subseteq W(B)$, то $W\left(\left[s_{1} \ldots s_{m} / C_{1} \ldots C_{m}\right](A)\right) \subseteq W\left(\left[s_{1} \ldots s_{m} / C_{1} \ldots C_{m}\right](B)\right)$.

$(33.27) W\left(S\left(C_{0}\right)\right) \subseteq W\left(S\left(\varphi_{0}\left(A_{0}\right)\right)\right)$ и $W\left(S\left(C_{0}\right)\right) \subseteq W\left(S\left(\varphi_{0}\left(B_{0}\right)\right)\right)$ (из $(33.2),(33.6),(33.17),(33.25)$ и $(33.26))$.

$(33.28) W\left(S\left(C_{0}\right)\right) \subseteq W\left(A_{0}\right)$ и $W\left(S\left(C_{0}\right)\right) \subseteq W\left(B_{0}\right)$ (из (33.22) и (33.27)).

(33.29) $W\left(S\left(C_{0}\right)\right) \subseteq W\left(A_{0}\right) \cap W\left(B_{0}\right)$ (из (33.28)).

(33.30) Существует такая $L$-формула $C$, что: $H \operatorname{Int}_{0, \omega} \vdash\left(A_{0} \supset\right.$ $C)$, Int $_{0, \omega} \vdash\left(C \supset B_{0}\right)$ и $W(C) \subseteq W\left(A_{0}\right) \cap W\left(B_{0}\right)$ (из того, что $S\left(C_{0}\right)$ есть $L$-формула, и утверждений (33.23) и (33.29)). Снимая 
допущение (33.1), получаем, что если $W\left(\varphi_{0}\left(A_{0}\right)\right) \cap W\left(\varphi_{0}\left(B_{0}\right)\right) \neq$ $\varnothing$, то существует такая $L$-формула $C$, что: $H \operatorname{Int}_{0, \omega} \vdash\left(A_{0} \supset C\right)$, $H$ Int $_{0, \omega} \vdash\left(C \supset B_{0}\right)$ и $W(C) \subseteq W\left(A_{0}\right) \cap W\left(B_{0}\right)$.

Утверждение (33) доказано.

(34) Существует такая $L$-формула $C$, что: $H \operatorname{Int} t_{0, \omega} \vdash\left(A_{0} \supset C\right)$, $H \operatorname{Int}_{0, \omega} \vdash\left(C \supset B_{0}\right)$ и $W(C) \subseteq W\left(A_{0}\right) \cap W\left(B_{0}\right)$ (из (32) и (33)). Снимая допущение $(21)$, получаем, что

(35) если существует такое $u$ из $\mathbf{N}$, что для некоторой пп $p$ $\left(\neg^{(u)} p\right)$ входит в $\left(A_{0} \supset B_{0}\right)$, то существует такая $L$-формула $C$, что: $\operatorname{HInt}_{0, \omega} \vdash\left(A_{0} \supset C\right), H \operatorname{Int}_{0, \omega} \vdash\left(C \supset B_{0}\right)$ и $W(C) \subseteq W\left(A_{0}\right) \cap$ $W\left(B_{0}\right)$.

(36) Существует такая $L$-формула $C$, что: $\operatorname{HInt}_{0, \omega} \vdash\left(A_{0} \supset C\right)$, $H \operatorname{Int}_{0, \omega} \vdash\left(C \supset B_{0}\right)$ и $W(C) \subseteq W\left(A_{0}\right) \cap W\left(B_{0}\right)$ (из (20) и (35)). Опираясь на $(36)$ и соглашение об обозначении, получаем, что

(37) существует такая $L$-формула $C$, что: $\left(A_{0} \supset C\right) \in$ Int $_{0, \omega}$, $\left(C \supset B_{0}\right) \in \operatorname{Int}_{0, \omega}$ и $W(C) \subseteq W\left(A_{0}\right) \cap W\left(B_{0}\right)$. Снимая допущения $(1)$ и $(2)$ и обобщая, получаем, что для всяких $L$-формул $A$ и $B$ : если $(A \supset B) \in \operatorname{Int}_{0, \omega}$ и $W(A) \cap W(B) \neq \varnothing$, то существует такая формула $C$, что $(A \supset C) \in \operatorname{Int}_{0, \omega},(C \supset B) \in \operatorname{Int}_{0, \omega}$ и $W(C) \subseteq W(A) \cap W(B)$.

Интерполяционная теорема для $\operatorname{Int}_{0, \omega}$ доказана.

Q.E.D.

\section{Литература}

[1] Попов В.М. Секвенциальные аксиоматизации простых паралогик //Логические исследования. Вып. 16. Центр гуманитарных инициатив, М.; СПб., 2010. C. 205-220.

[2] Смирнов B.A. Формальный вывод и логические исчисления. М., 1972.

[3] Шютmе K. Интерполяционная теорема для интуиционистской логики предикатов //Математическая теория логического вывода. М., 1967. С. 285-295.

[4] Craig W. Linear reasoning. A new form of the Herbrand-Gentzen theorem //Journal of Symbolic Logic. 1957. Vol. 22. P. 250-268.

Замечание. В публикации В.М. Попова «Секвенциальная аксиоматизация паранормальной логики PContPComp» (Логические исследования. Вып. 17. С. 240-245) на стр. 243-244 в формулировке правила ВОКЛ, в формулировке правила ВИП и в формулировке ВОИП имеются опечатки. Правильные формулировки этих правил таковы: 


$$
\begin{aligned}
& \frac{((\neg A) \bullet \Gamma) \rightarrow \Theta \quad((\neg B) \bullet \Gamma) \rightarrow \Theta}{((\neg(A \& B)) \bullet \Gamma) \rightarrow \Theta}(\text { ВОКЛ }) \\
& \frac{(A \bullet \Gamma) \rightarrow(\Theta \bullet B)}{\Gamma \rightarrow(\Theta \bullet(A \supset B))}(\text { ВИП }) \\
& \frac{\Gamma \rightarrow(\Theta \bullet A) \quad \Gamma \rightarrow(\Theta \bullet(\neg B))}{\Gamma \rightarrow(\Theta \bullet(\neg(A \supset B)))}(\text { ВОИП })
\end{aligned}
$$

В публикации В.М. Попова «Секвенциальные аксиоматизации пропозициональных логик нельсоновского типа» (Логические исследования. Вып. 17. С. 246-250) допущены ошибки:

(1) утверждение о том, что для исчисления $G P \operatorname{Cont}(N)$ верна теорема об устранимости сечения,

(2) утверждение о том, что позитивный фрагмент логики $P \operatorname{Cont}(N)$ равен позитивному фрагменту интуиционистской пропозициональной логики,

(3) утверждение о том, что логика $P \operatorname{Cont}(N)$ не имеет конечной характеристической матрицы.

В исчислении $G P \operatorname{Cont}(N)$ сечение неустранимо, позитивный фрагмент логики $P \operatorname{Cont}(N)$ равен позитивному фрагменту классической (а не интуиционистской) пропозициональной логики. Логика $\operatorname{PCont}(N)$ имеет конечную характеристическую матрицу, поскольку, как показал В.О. Шангин, $P \operatorname{Cont}(N)$ равна трехзначной логике, являющейся множеством всех теорем исчисления PCont, построенного в работе Л.И. Розоноэра «О выявлении противоречий в формальных теориях.1» (Автоматика и телемеханика. № 6,1983$)$. 